\section{Resultados da assistência ao parto no Centro de Parto Normal Dr. David Capistrano da Costa Filho em Belo Horizonte, Minas Gerais, Brasil}

\author{
Results of childbirth care at a birthing center in \\ Belo Horizonte, Minas Gerais, Brazil
}

\footnotetext{
1 Hospital Sofia Feldman, Belo Horizonte, Brasil.

${ }^{2}$ Faculdade de Saúde e Ecologia Humana, Vespasiano, Brasil.

3 Escola de Enfermagem, Universidade Federal de Minas Gerais, Belo Horizonte, Brasil.

Correspondência S. E. V. Campos Hospital Sofia Feldman. Rua Antônio Bandeira 1060 Belo Horizonte, $M G$ 31840-130, Brasil. sibyllecampos@hotmail.com
} 
obstétrica atual, altamente intervencionista, que trata todas as gestações e partos como se fossem de risco ${ }^{2}$.

Desde o primeiro CPN, fundado em 1975 pela Maternity Center Association em Nova York, Estados Unidos, observa-se um crescimento constante dessa modalidade assistencial no mundo inteiro. Difundiu-se o discurso acerca das vantagens de um local de parto, fora do hospital, acolhedor e similar ao domicílio, porém com equipamentos e infra-estrutura técnica e recursos humanos adequados para as mulheres com gestação de baixo risco 3,4,5.

Apesar de, no Brasil, a assistência em CPN ser uma modalidade inovadora, existem estudos internacionais mostrando resultados maternos e neonatais favoráveis sobre a assistência em casas de parto $6,7,8,9,10,11,12,13,14,15,16,17$. Hodnett 18 encontrou diferenças significativas entre os resultados referentes a alguns indicadores de processo da assistência. Segundo a meta-análise do autor, que incluiu somente estudos randomizados, mulheres atendidas em casas de parto utilizaram em menor extensão métodos farmacológicos para o alívio da dor e ocitocina para a aceleração do trabalho de parto. Uma menor quantidade de mulheres foi submetida à episiotomia e impedida de se movimentar e deambular durante o trabalho de parto. Foram observadas anormalidades na freqüência cardíaca fetal em menor número, assim como uma incidência menor de partos cirúrgicos. A satisfação das mulheres foi maior com o atendimento nas casas de parto. Hodnett 18 alerta para uma tendência maior, mas não significativa, para a mortalidade perinatal no CPN, devido à inclusão de estudos com randomização na gestação.

Existem, hoje, em Minas Gerais, dois Centros de Parto: um na modalidade extra-hospitalar e o CPN estudado, na modalidade intra-hospitalar. O CPN Dr. David Capistrano da Costa Filho funciona de acordo com a Portaria no. 985/1999 1, integrado ao hospital Sofia Feldman, porém preservando a autonomia do profissional enfermeiro, respaldado pela Lei $n^{o}$. 7.498, que regulamenta o exercício da enfermagem no país 19.

Sem resultados nacionais disponíveis sobre a assistência em CPN, é possível, por enquanto, orientar-se somente em estudos internacionais. A discussão emergente sobre a segurança da assistência ali prestada torna necessária uma avaliação objetiva dos resultados dessa modalidade de assistência. O presente estudo visa à descrição e discussão dos resultados dos principais indicadores clínicos da assistência prestada num CPN que atende, ao lado do seu hospital de referência, as parturientes de baixo risco na periferia de Belo Horizonte.

\section{Material e método}

Foi realizado um estudo retrospectivo da assistência ao parto de 2.117 mulheres admitidas no CPN Dr. David Capistrano da Costa Filho em Belo Horizonte, no período de janeiro de 2002 a julho de 2003, utilizando como fonte dados obtidos no sistema de informação interno, no livro de registro da unidade e nos prontuários individuais em caso de inconsistência entre as fontes ou de inexistência dos dados. O CPN referido foi inaugurado em setembro de 2001, funcionando plenamente a partir de janeiro 2002, data do início desta pesquisa. O objetivo da coleta de dados foi determinado por questões operacionais relacionadas com o curso de Mestrado.

Entre as variáveis selecionadas, constam: taxa de transferência materna e suas causas; taxa de cesárea e parto vaginal assistido; percentual de avaliações pelo médico obstetra e suas causas; taxa de valor de Apgar no 1o e 5 o minutos de vida; taxa de admissão em unidade neonatal e suas causas; percentual das avaliações pelo pediatra e suas causas; e mortalidade neonatal. Os dados foram trabalhados com programa Epi Info 6.1 (Centers for Disease Control and Prevention, Atlanta, Estados Unidos), a partir de análise de freqüências.

O estudo foi apresentado ao Comitê de Ética da Universidade Federal de Minas Gerais e do Hospital Sofia Feldman atendendo, a Resolução $n^{o}$. 196/96 do Conselho Nacional de Saúde, que dispõe sobre princípios éticos em pesquisas envolvendo seres humanos. A coleta de dados foi iniciada somente após a aprovação da pesquisa nessas instituições.

\section{Local do estudo}

O CPN Dr. David Capistrano da Costa Filho assiste cerca de cem partos por mês, dispondo de cinco unidades pré-parto, parto e pós-parto, e da possibilidade do parto na água. Ele é situado diretamente ao lado do hospital da sua referência, o Hospital Sofia Feldmann. Ambas as unidades fazem parte da Fundação de Assistência Integral à Saúde, uma instituição privada com missão filantrópica, que atende o usuário do Sistema Único de Saúde (SUS). A equipe diária é composta por uma enfermeira obstetra com qualificação em reanimação neonatal, um auxiliar de enfermagem e um agente administrativo. Os demais serviços são compartilhados com seu hospital de referência.

De acordo com a Portaria no. 985/1999 1 cada CPN tem de dispor de um protocolo assistencial, que servindo de manual técnico, estabelece os 
critérios da admissão da parturiente, as condutas a serem tomadas durante a assistência no CPN e as condições clínicas da parturiente e do recém-nascido, que determinam a transferência de ambos no caso de intercorrências e desvios da evolução fisiológica.

Como os dados foram coletados retrospectivamente, e na fonte para coleta dos dados não está prevista a inclusão da informação sobre o desejo expresso das mulheres em serem atendidas no CPN, não foi possível identificar os dois grupos. Portanto, não foram discriminados os resultados de acordo com a inclusão no grupo com conhecimento prévio e desejo expresso ou no grupo sem conhecimento prévio.

Os critérios de admissão para assistência no CPN incluem: gestação entre 37 e 41 semanas completas, fase ativa do trabalho de parto, apresentação fetal de vértice, e ausência de mecônio, macrosomia fetal ou COUR, ou anormalidades nos batimentos cardíacos fetais. A parturiente deve ser afebril e normotensa, sem histórico de cesárea anterior ou rotura de membranas com duração maior do que seis horas, e sem qualquer intercorrência clínica ou obstétrica. A transferência materna é obrigatória em casos de detecção de líquido meconial; desacelerações cárdio-fetais, indicando estado fetal não-tranqüilizador; parada de progressão do trabalho de parto por duas horas com atividade uterina adequada; hipertonia ou hiperdinamismo uterino; hemorragias durante ou após o parto; lacerações de $3^{\circ} \mathrm{e} 4^{\circ}$ graus; retenção placentária; hematomas da parede vaginal; elevação da pressão arterial acima ou igual a 140/90mmHg; e febre durante ou após o parto. Também deve ser removido o recém-nascido submetido a manobras de ressuscitação e que permanece instável hemodinamicamente; o recém-nascido com peso abaixo de $2.500 \mathrm{~g}$ ou pré-termo; e o recém-nascido com qualquer intercorrência clínica, incluindo suspeita de malformação congênita, quadro infeccioso e desconforto respiratório.

A assistência prestada no CPN estudado apóia-se no modelo humanista e resgata as características fisiológicas e naturais do nascimento. $\mathrm{O}$ parto é considerado uma vivência emocional da mulher e um evento social da família; como conseqüência, a assistência é centrada na mulher, na sua família e em suas necessidades biopsicossociais 20,21. Portanto, o atendimento deve ser individualizado e flexível, com apoio emocional contínuo e transmitindo à mulher a sensação de ser compreendida. As intervenções, que permitem discussão e decisão compartilhada, devem ser adotadas após o consentimento da mulher, assegurando à parturiente um papel ativo e de controle do processo de nascimento. Deve ser encorajada a presença de acompanhantes, de acordo como o desejo da mulher. Suas vontades em relação à movimentação e posições para o segundo estágio do parto são respeitadas, assim como suas emoções, sem julgamento algum. O recém-nascido é colocado em cima do ventre da mãe, em contato pele a pele, assim que nasce, e permanece com ela o tempo todo.

É permitida a utilização de ocitocina e da amniotomia para correção de hipodinamismo primário e a realização de episiotomia, quando for necessário, assim como a episiorrafia e a sutura de lacerações de 1o e 2 o graus pela enfermeira obstetra, sob anestesia local. A monitorização do bem-estar fetal é realizada com auscultação intermitente e a evolução do trabalho de parto é acompanhada por meio do registro no partograma, editado pelo Centro Latino-Americano de Perinatologia e Desenvolvimento Humano - CLAP 22. A assistência é prestada de acordo com as evidências científicas existentes para a assistência ao parto normal, incluindo a deambulação durante o trabalho de parto, a escolha da posição para o 2 o estágio de parto pela mulher, a hidratação oral, assim como o uso de meios não-farmacológicos para o alívio da dor, como massagens e banhos 2 .

Tanto a assistência à mulher como ao recémnascido é inteiramente realizada pela enfermeira obstetra, incluindo a alta hospitalar, com 12 a 24 horas. A proximidade com o Hospital Sofia Feldman facilita a presença do obstetra ou pediatra, quando solicitada.

\section{Resultados}

Entre as 2.117 mulheres admitidas no CPN entre janeiro 2002 e julho 2003, duas tiveram alta por falso trabalho de parto. Das mulheres admitidas no CPN, 25,8\% tinham até 19 anos, inclusive, e $4,9 \%$ tinham acima de 35 anos. Cerca da metade das mulheres, $48,8 \%$, foram nulíparas e a maior parcela, $45,2 \%$, foi constituída de mulheres que não concluíram o primeiro grau do ensino fundamental.

A Tabela 1 mostra as taxas dos indicadores de assistência em relação ao total das mulheres admitidas no CPN (2.117). A taxa de transferência é de $11,4 \%$, sendo 10,5\% transferências intraparto e $0,9 \%$ ocorrida no período pós-parto. Foram transferidas $16 \%$ das nulíparas e $7 \%$ das multíparas, i.e., das mulheres com um ou mais partos anteriores.

Das mulheres transferidas durante o trabalho de parto, $63 \%$ foram assistidas pelas enfermeiras obstétricas de plantão no hospital de referência. Os partos das demais mulheres transferidas 
Indicadores assistenciais das mulheres admitidas no Centro de Parto Normal (CPN) e dos seus recém-nascidos.

\begin{tabular}{|c|c|c|}
\hline Indicadores & $\mathbf{n}$ & $\%$ \\
\hline \multicolumn{3}{|l|}{ Maternos } \\
\hline \multicolumn{3}{|l|}{ Tipo de parto } \\
\hline Normal & 2.051 & 96,9 \\
\hline Cesárea & 46 & 2,2 \\
\hline Fórceps & 18 & 0,8 \\
\hline Sem dados (alta por falso trabalho de parto) & 2 & 0,1 \\
\hline Transferência materna & 241 & 11,4 \\
\hline Solicitação da presença do médico obstetra & 42 & 2,0 \\
\hline \multicolumn{3}{|l|}{ Neonatais } \\
\hline \multicolumn{3}{|l|}{ Admissão no CPN } \\
\hline Sem admissão em Unidade Neonatal & 2.035 & 96,2 \\
\hline Com admissão em Centro de Terapia Intensiva & 26 & 1,2 \\
\hline Com admissão em Unidades de Cuidados Intermediários & 54 & 2,6 \\
\hline \multicolumn{3}{|l|}{ Apgar no 1 o minuto } \\
\hline$>7$ & 2.027 & 95,8 \\
\hline$<7$ & 87 & 4,1 \\
\hline Sem dados & 3 & 0,1 \\
\hline \multicolumn{3}{|l|}{ Apgar no 5 o minuto } \\
\hline$>7$ & 2.093 & 98,9 \\
\hline$<7$ & 21 & 1,0 \\
\hline Sem dados & 3 & 0,1 \\
\hline Mortalidade neonatal e pós-neonatal corrigida & 4 & 0,2 \\
\hline
\end{tabular}

$\mathrm{N}$ total $=2.117$

Fonte: Prontuários/Centro Latino-Americano de Perinatologia e Desenvolvimento Humano.

foram realizados por médico obstetra, via vaginal ou via abdominal.

Foram listadas, na Tabela 2, as causas para a transferência intraparto: trabalho de parto prolongado e desejo de analgesia, com 25,8\% e $25,3 \%$, respectivamente; presença de líquido meconial detectada após a admissão da parturiente, com 18,7\%; seguida de alterações dos batimentos cárdio-fetais, com $8,7 \%$, que indicam um estado fetal não intermediário e um possível sofrimento fetal. Com freqüência, o exame clínico-tocológico não é repetido imediatamente após a admissão no CPN pela enfermeira, assim como os resultados dos exames laboratoriais não estão disponíveis imediatamente após a admissão. Fatos como os apontados levaram à admissão de mulheres que não atendiam os critérios de gestação de baixo risco em 13 casos, ou 5,4\%, indicados como "outros" na Tabela 2. Treze mulheres desenvolveram, durante sua estada no CPN, quadros clínicos com elevação de pressão arterial igual ou maior que $140 / 90 \mathrm{mmHg}$, e sete mulheres $(2,9 \%)$ apresentaram picos febris maiores ou iguais a $38^{\circ}$, tornando-se parturientes ou puérperas de risco, sendo, conseqüentemente, referidas para o hospital. Houve, ainda, dois casos de prolapso de cordão $(0,8 \%)$, ambos resolvidos satisfatoriamente no hospital de referência, e dois casos de apresentação anômala, detectados somente durante o trabalho de parto no CPN e transferidos antes do parto. Entre as causas da transferência pós-parto de vinte mulheres, destaca-se a hemorragia, com 4,6\%. Houve transferência para sutura de lacerações de 3 o grau em dois casos.

A modalidade de unidade intra-hospitalar facilita a colaboração entre o CPN e os médicos obstetras e pediatras. A presença dos médicos obstetras foi solicitada para 41 , ou $2,2 \%$, das mulheres não transferidas antes ou após o parto ( $\mathrm{n}=1.876)$, como mostra a Tabela 1 . De acordo com a Tabela 3, a principal causa foi a assistência ao parto, em $30,9 \%$ das mulheres assistidas no CPN, e a reparação de lacerações severas, em $26,2 \%$ dos casos. Somando-se a solicitação da presença do médico para os casos de hemorragia pós-parto em oito mulheres com as 11 mulheres que foram transferidas por esta causa, evidencia- 
Distribuição das causas de transferência materna.

\begin{tabular}{|c|c|c|}
\hline Causas de transferência & $\mathbf{n}$ & $\%$ \\
\hline \multicolumn{3}{|l|}{ Intraparto } \\
\hline Distócias de trabalho de parto & 62 & 25,8 \\
\hline Desejo por analgesia & 61 & 25,3 \\
\hline Líquido meconial & 45 & 18,7 \\
\hline Estado fetal não-tranqüilizador & 21 & 8,7 \\
\hline Pressão arterial $\geq 140 / 90 \mathrm{mmHg}$ & 9 & 3,7 \\
\hline Febre & 4 & 1,7 \\
\hline Hemorragia & 2 & 0,8 \\
\hline Apresentação anômala & 2 & 0,8 \\
\hline Prolapso de cordão & 2 & 0,8 \\
\hline Outros & 13 & 5,4 \\
\hline \multicolumn{3}{|l|}{ Pós-parto } \\
\hline Hemorragia & 11 & 4,6 \\
\hline Pressão arterial $\geq 140 / 90 \mathrm{mmHg}$ & 4 & 1,7 \\
\hline Febre & 3 & 1,2 \\
\hline Laceração de 3o grau & 2 & 0,8 \\
\hline Total & 241 & 100,0 \\
\hline
\end{tabular}

Fonte: Prontuários/Centro Latino-Americano de Perinatologia e Desenvolvimento Humano.

Tabela 3

Distribuição das causas para solicitação da presença do médico obstetra.

\begin{tabular}{lcc}
\hline Causas & $\mathbf{n}$ & $\%$ \\
\hline Assistência ao parto & 13 & 30,9 \\
Sutura de laceração & 11 & 26,2 \\
Hemorragia pós-parto & 8 & 19,1 \\
Retenção placentária & 4 & 9,5 \\
Outras & 5 & 12,9 \\
Sem informação & 1 & 2,4 \\
Total & 42 & 100,0 \\
\hline
\end{tabular}

Fonte: Prontuários/Centro Latino-Americano de Perinatologia e Desenvolvimento Humano.

se um ponto fraco da assistência no CPN estudado. Houve 19 casos de hemorragia pós-parto, ou $0,9 \%$ das mulheres admitidas no CPN, porcentagem maior do que a relatada na literatura 14,15,17.

De acordo ainda com a Tabela 1, a taxa de cesárea entre as mulheres admitidas no CPN (2.117 mulheres) foi de $2,2 \%$, e a taxa de parto a fórceps $0,8 \%$. Dos partos a fórceps, cinco foram passados no próprio $\mathrm{CPN}$, após solicitação da presença do médico obstetra.
Em relação aos indicadores dos recém-nascidos, a Tabela 1 mostra os valores de Apgar dos recém-nascidos, assim como a taxa de transferência neonatal para o CTI e para a unidade de cuidados intermediários. Entre os recém-nascidos das mulheres admitidas no CPN, incluindo os das mulheres transferidas, $4,1 \%$ receberam um valor de Apgar abaixo de 7 no lo minuto e $1 \%$ no 5o minuto. Do total das mulheres admitidas no CPN, 80 (3,8\%) recém-nascidos foram admitidos em unidade neonatal, resultando numa taxa de admissão em Centro de Tratamento Intensivo (CTI) de 1,2\% e de 2,6\% na unidade de cuidados intermediários.

Houve seis mortes neonatais e pós-neonatais de recém-nascidos cujas mães foram admitidas no CPN em trabalho de parto, sendo uma morte referente a uma transferência materna. Nesse caso, a parturiente foi transferida com batimentos cárdio-fetais dentro da normalidade, e depois de ter permanecido durante uma hora no CPN após a presença de líquido meconial ter sido detectada. Foi realizada uma cesárea de urgência, por possível sofrimento fetal, após seis horas em trabalho de parto na maternidade. Um recém-nascido nasceu com Apgar 7/8, sem intercorrências durante o trabalho de parto e parto. Ele desenvolveu apnéias consecutivas, com melhora após estimulações táteis, e foi transferido imediatamente para o CTI, com oxigênio 
sob cateter nasal. Apresentou piora do padrão respiratório e faleceu após dois meses. Discutiuse uma patologia sindrômica sem confirmação. Outro recém-nascido do CPN, Apgar 7/9, faleceu após uma transferência para o CTI com três horas de vida, por desconforto respiratório. Um recém-nascido, Apgar 4/8, cuja mãe teve história de toxoplasmose sem tratamento e polidrâmnio, foi transferido para o CTI. Inicialmente estável, o quadro apresentado pelo recém-nascido piorou posteriormente, talvez após aspiração de dieta. Seu exame clínico sugeriu hidronefrose e hidrocefalia. Dois recém-nascidos tinham malformações congênitas cardíacas severas, resultando numa taxa corrigida de mortalidade neonatal de 2 por mil nascidos vivos.

Como mostra a Tabela 4, que se refere aos indicadores dos recém-nascidos do CPN, 3,2\% dos recém-nascidos que nasceram no CPN receberam um valor de Apgar abaixo de sete no 1o minuto, e $0,7 \%$ no 5o minuto. Dos recém-natos com valor de Apgar menor do que 7 no lo minuto de vida, $75 \%$ foram reanimados com eficácia pela enfermeira obstetra, recebendo um valor de Apgar menor do que 7 no 5o minuto de vida. Dos recém-nascidos no CPN, 63 necessitaram de cuidados especiais, correspondendo a uma taxa de admissão em CTI de 0,9\%, e de 2,4\% em unidade de cuidados intermediários.

Analisando-se as taxas de Apgar menor do que 7 e as taxas de transferência neonatal tanto para o CTI quanto para unidade de cuidados intermediários, entre os recém-nascido das mães transferidas e das mães cujos partos foram assistidos no CPN, evidencia-se uma diferença significativa. Os recém-nascidos das mães transferidas tiveram um risco relativo de 5.28 (2.21< $\mathrm{RR}<12.6$; IC95\%) para um valor de Apgar menor do que 7 no 5 o minuto, um risco relativo de 4.54 $(2.05<\mathrm{RR}<10.07)$ especificamente para a admissão em CTI e um risco relativo de 2.42 (1.34 $>\mathrm{RR}>4.35$; IC95\%) para transferência para as unidades neonatais. Apesar de todos os resultados apresentarem significância estatística, o intervalo extenso do risco relativo indica limitações pela incidência baixa dos eventos, exceto para a última variável, que soma as admissões em CTI e em unidade de cuidados intermediários e apresenta uma incidência maior.

Do total das mulheres admitidas no CPN (2.117), oitenta tiveram partos em que os recém-nascidos foram encaminhados às unidades de atenção neonatal no hospital de referência, conforme mencionado na Tabela 1. Dentre as causas para essas transferências, conforme Tabela 5, destaca-se como principal a depressão ou desconforto respiratório com $36,3 \%$ das admissões, incluindo os recém-nascidos das mulheres transferidas e também não-transferidas. A segunda causa é o suspeito ou infecção instalada com $22,5 \%$, seguida de icterícia com $18,8 \%$. Esse achado está de acordo com a literatura consultada e mostra a vulnerabilidade do recém-nascido na hora do nascimento 10,15,17.

Dos recém-nascidos que não foram transferidos, $178(9,4 \%)$, foram avaliados pelo pediatra

Tabela 4

Indicadores assistenciais dos recém-nascidos do Centro de Parto Normal (CPN).

\begin{tabular}{lcc}
\hline Indicadores & $\mathbf{n}$ & $\%$ \\
\hline Admissão no CPN & & 96,7 \\
Sem admissão em Unidade Neonatal & 1.833 & 0,9 \\
Com admissão Centro de Tratamento Intensivo & 17 & 2,4 \\
Com admissão em Unidade de Cuidados Intermediários & 46 & 96,7 \\
Apgar no 1o minuto & 1.835 & 3,0 \\
$>7$ & 60 & 0,1 \\
$<7$ & 1 & 99,2 \\
Sem dados & & 0,7 \\
Apgar no 5o minuto & 1.882 & 0,1
\end{tabular}

$\mathrm{N}$ total $=1.896$.

Fonte: Prontuários/Centro Latino-Americano de Perinatologia e Desenvolvimento Humano. 
Distribuição das causas da admissão em unidade neonatal.

\begin{tabular}{lcc}
\hline Causas da admissão & $\mathbf{n}$ & $\%$ \\
\hline Depressão/Desconforto respiratório & 29 & 36,3 \\
Infecção & 18 & 22,5 \\
Icterícia & 15 & 18,8 \\
Anomalias congênitas & 5 & 6,3 \\
Prematuridade & 4 & 5,0 \\
Pequeno para idade gestacional/grande para idade gestacional & 3,7 \\
Hipotonia/Sucção débil & 3 & 2,5 \\
Outras & 2 & 3,7 \\
Sem dados & 3 & 1,2 \\
Total & 1 & 100,0 \\
\hline
\end{tabular}

Fonte: Prontuários/Centro Latino-Americano de Perinatologia e Desenvolvimento Humano.

por diversas causas, como analisado na Tabela 6 . Quatorze recém-nascidos do CPN $(0,7 \%)$ foram assistidos no parto pelo pediatra, revelando, por um lado, a colaboração do profissional médico no modelo intra-hospitalar e, por outro, a limitação do enfermeiro nesses casos.

Como mostra a Tabela 4, avaliação pelo pediatra ocorreu em 9,4\% dos recém-nascidos que nasceram no CPN e que não foram transferidos, correspondendo a 178 recém-nascidos. Segundo a Tabela 6, uma parcela importante desse total - 19\% dos recém-nascidos avaliados - foi encaminhada para avaliação em razão de dúvidas levantadas pelo enfermeiro durante o exame físico, que não se confirmaram como anormalidades. Isso reflete, ainda, uma certa insegurança do enfermeiro em relação à avaliação do recém-nascido, tendência que deve diminuir na medida em que cresce a sua experiência no procedimento. O desconforto respiratório foi responsável pela avaliação pelo pediatra em 15,2\% dos casos, mostrando, uma vez mais, a importância desta afecção. Anomalias congênitas menores e recém-nascidos apresentando medidas não condizentes com a idade gestacional, mas estáveis e com boa sucção, foram as causas da avaliação em 10,7\% e 9,5\%, respectivamente. A assistência do pediatra no parto foi solicitada em 7,9\% dos casos, refletindo uma limitação da assistência do enfermeiro. O item "outras" $(19,1 \%)$ inclui avaliações solicitadas por presença de ruídos cardíacos, ocorrência de náusea/vômitos, fratura de clavícula, valores de Apgar baixo ao nascer, e uma lesão de plexo braquial.

\begin{tabular}{|c|c|c|}
\hline \multirow{2}{*}{\multicolumn{3}{|c|}{$\begin{array}{l}\text { Distribuição das causas de avaliação dos recém-nascidos no } \\
\text { Centro de Parto Normal pelo pediatra. }\end{array}$}} \\
\hline & & \\
\hline Causa de avaliação pelo pediatra & $\mathbf{n}$ & $\%$ \\
\hline \multicolumn{3}{|l|}{ Dúvidas no exame físico do } \\
\hline recém-nato não confirmado & 34 & 19,1 \\
\hline Desconforto respiratório & 27 & 15,2 \\
\hline Anomalias congênitas menores & 19 & 10,7 \\
\hline \multicolumn{3}{|l|}{ Pequeno para idade gestacional/ } \\
\hline Grande para idade gestacional & 17 & 9,5 \\
\hline Assistência ao parto & 14 & 7,9 \\
\hline Hipotonia/Sucção débil & 11 & 6,2 \\
\hline Suspeita de infecção & 10 & 5,6 \\
\hline Icterícia & 6 & 3,4 \\
\hline Prematuridade & 2 & 1,1 \\
\hline Outras & 34 & 19,1 \\
\hline Sem dados & 4 & 2,2 \\
\hline Total & 178 & 100,0 \\
\hline
\end{tabular}

Fonte: Prontuários/Centro Latino-Americano de Perinatologia e Desenvolvimento Humano. 


\section{Discussão}

Na discussão dos resultados, deve-se ter em mente que o CPN em estudo estava há pouco tempo em funcionamento quando foram coletados os dados. Sua implantação constituiu uma experiência inovadora tanto para os enfermeiros obstétricos do CPN quanto para a equipe do hospital de referência. Alguns resultados podem ter sido influenciados por essa circunstância.

Em primeira mão, este estudo levantou dados da assistência ao parto de baixo risco inexistentes na literatura nacional. A limitação do estudo deve-se ao seu delineamento descritivo, que não permite comparação, à inexistência de dados nacionais para a população estudada e ao tamanho insuficiente da amostra para calcular a mortalidade perinatal. A comparação dos dados do presente estudo com dados de Centros de Parto nos Estados Unidos e na Europa foi prejudicada pela grande diversidade da estrutura, dos critérios de seleção da clientela e das rotinas empregadas na assistência em casas de parto.

Contudo, percebe-se, na discussão dos resultados, que eles não se diferenciam dos resultados das pesquisas internacionais $6,7,8,9,10,11,12,13$, $14,15,16,17$, todos considerados positivos pelo meio acadêmico. A taxa de cesariana de $2,2 \%$, quando comparada com estudos internacionais com taxas entre $0,8 \%$ e $7,1 \%$ 7,8,10,15,16,17, constitui um resultado positivo, bem abaixo da média nacional e dos valores recomendados pela OMS, em torno de $10 \%$ a $15 \% 23$. Visto que a taxa de cesárea nas instituições, que atendem pelo SUS, em 2000 foi em torno de $24 \% 20$, a taxa alcançada no estudo mostra que a assistência no CPN, como estratégia para reduzir a taxa de cesárea, é eficaz. Os estudos comparativos na literatura entre grupos de mulheres admitidas em Casa de Parto e grupos de controle encontraram, entre os grupos, não somente menores taxas de intervenções, inclusive taxa de cesáreas, como também maior satisfação materna com a assistência e os resultados perinatais, que não diferem entre os grupos $6,7,8,12,13,14,17$.

Uma análise mais detalhada das transferências e dos casos, nos quais a realização de uma cesárea ou de um parto vaginal assistido se tornou necessária, e a realização de um estudo caso-controle poderiam responder se houve ou não inoportunidade de assistência no CPN. Apesar de que a diferença na taxa de cesárea e na admissão no CTI entre o grupo de mulheres que foi transferido e o que não foi transferido é significativa, não se pode apontar a assistência no CPN ou a demora na transferência como determinantes da diferença. A metodologia aplicada neste estudo não permite essa análise. Cinqüenta por cento das mulheres foram transferidas por desejo de analgesia e trabalho de parto prolongado já estabelecido. Em 27,4\% das transferências houve uma possível limitação no bem-estar do feto, como alterações nos batimentos cárdio-fetais e a ocorrência de líquido meconial durante o trabalho de parto. Todos os fatores podem levar comprovadamente a resultados negativos. Para poder chegar a um resultado conclusivo nessa questão, deveria se lançar mão de uma metodologia analítica com estudos do tipo casocontrole.

Não foi possível relacionar os resultados de acordo com o conhecimento prévio e a escolha consciente da parturiente para esta modalidade de assistência. Os resultados positivos da assistência em casa de parto são freqüentemente associados à aceitação e maior motivação da parturiente para um parto sem intervencionismo. A continuidade de assistência desde o pré-natal até o pós-parto mediato, e o atendimento com razão profissional/parturiente 1:1 ou em dimensões semelhantes são estratégias importantes adotadas pelas casas de parto no mundo inteiro e sua influência sobre os resultados perinatais é clara 24,25 . O centro de parto estudado não oferece continuidade de assistência abrangendo o período pré-natal e o trabalho de parto, pois os profissionais trabalham em regime de plantão. Oferece somente uma assistência não massificante, com cada profissional cuidando, no máximo, de três mulheres em trabalho de parto. Tanto a ausência da continuidade como a baixa razão profissional/parturiente podem ter influenciado o resultado. Somente estudos com regressão logística e multifatoriais podem discernir a influência de cada fator.

A taxa de transferência materna não pode ser vista como um indicador de qualidade da assistência. Uma pergunta importante a se fazer, seria, se as transferências maternas, quando realizadas precocemente, não poderiam ter melhorado os indicadores perinatais, uma vez que a taxa é baixa no CPN estudado. O estudo realizado não pode responder devido à limitação metodológica. Mais uma vez se impõe a necessidade de estudos do tipo caso-controle. Atingindo 11,4\% das mulheres admitidas no CPN em estudo, ela pode ser considerada baixa quando comparada com os estudos internacionais e mostra a resolutividade do CPN em estudo. As taxas podem, ainda, ser reduzidas quando não se admitem primigestas rotineiramente $6,11,16$. A maioria dos estudos mostra taxas mais altas, entre $16 \%$ e $30 \%$ 8,9,10,12,15,17,20. Evidenciam-se as vantagens da unidade intrahospitalar, onde solicitações do pediatra e do obstetra podem substituir transferências, que se impõem em unidades extra-hospitalares. As 
taxas de avaliação pelo obstetra e pelo médico devem ser analisadas nesse aspecto.

Uma das principais causas da transferência - o trabalho de parto prolongado - revela uma contradição, já que, no CPN em estudo, o uso da ocitocina para correções de hipodinamismo uterino é permitido, levantando a necessidade de discussão deste fato. Na literatura, distócias de trabalho de parto também são citadas como causa principal na maioria dos estudos. O desejo de analgesia como outra causa importante para transferência, revela o fato de que cerca de $25 \%$ das mulheres admitidas no CPN não suportam o trabalho de parto sem analgesia. Na literatura, o desejo de analgesia como causa da transferência é referido em taxas mais variadas. Além de variações na oferta de meios farmacológicos nos CPN citados na literatura, uma questão cultural, que implica a aceitação da dor de parto pela mulher, deve interferir na taxa de transferência por desejo de analgesia entre os CPN em comparação.

$\mathrm{O}$ percentual dos recém-nascidos no CPN que receberam um valor de Apgar no 5o minuto abaixo de 7 é ligeiramente superior ao relatado na maioria dos estudos 9,10,15,16,17.

A taxa de admissão em CTI neonatal de 1,2\% entre o total das mulheres admitidas em CPN e 0,9\% entre os recém-nascidos no CPN estudado, é um resultado positivo e semelhante ao estudo de Greulich et al. 10, que relatam uma taxa de cesárea de $1,8 \%$, taxa de admissão em CTI neonatal de $1,5 \%$ e nenhuma morte intraparto fetal ou materna. A população do estudo referenciado apresentava características sócio-demográficas semelhantes às da população estudada no presente trabalho e a grande maioria das parturientes não tinha contato prévio com a instituição. Cunningham et al. 27 também relatam resultados perinatais excelentes num centro de parto intra-hospitalar do Parkland Hospital, com uma taxa de mortalidade corrigida de $0,2 /$ mil e taxa de cesárea de 4,7\%. Em relação a outros estudos internacionais, que relatam taxas de admissão em CTI neonatal entre $1 \%$ e $9,7 \% 13,14$, a taxa de admissão em CTI no CPN estudado é menor. A grande variação entre as taxas indica diferenças nos critérios de admissão, o que dificulta uma comparação mais consistente. Uma tendência favorável para os resultados do CPN em estudo é evidente.

O presente estudo não é conclusivo acerca da mortalidade neonatal, pois o tamanho da amostra é demasiadamente pequeno em relação à incidência da variável. Estudos descritivos citam taxas de 2 a 4/mil 8,9,13. Greulich et al. 10 e Cunningham et al. 26 , relatam excelentes taxas de mortalidade perinatal dos centros de parto intrahospitalar, com estudos incluindo populações de 36.410 e 20.047 mulheres, respectivamente. Analisando as seis mortes neonatais registradas no estudo, percebe-se que, em dois casos, a assistência no CPN poderia ser caracterizada como inoportuna. Nos demais casos, aparentemente não houve falha na assistência no CPN.

Apesar da metodologia utilizada não permitir conclusões sobre a qualidade da assistência em CPN, poderia se discutir os desfechos negativos. Entre o total das mulheres admitidas no CPN, na pior das hipóteses, 86 casos poderiam ser considerados como desfechos negativos: oitenta admissões em unidade neonatal e seis mortes neonatais, totalizando 3,5\%. É importante para a discussão, que essa porcentagem inclui admissões por causas não dependentes da assistência como malformações, icterícia, recém-nascidos com peso inadequado para idade gestacional e admissões em unidade de cuidados neonatais intermediários. Além disso, as mulheres transferidas, as quais apresentaram incidência maior de admissão em unidade neonatal, receberam a assistência na maternidade e esta poderia também ter contribuído para os desfechos negativos. Entre gestações de baixo risco, todos os desfechos negativos são evitáveis a não ser aqueles resultantes de má-formação congênita. Em nível nacional não existem dados referentes a resultados desejáveis e/ou aceitáveis em gestações de baixo risco. Assim, para fins de avaliação, essa taxa somente pode ser comparada, ainda com limitações, com dados na literatura. A porcentagem de $3,5 \%$ não difere dos estudos internacionais e poderia até servir como base para discussão em nível nacional.

\section{Conclusão}

Considerando estudos internacionais, os indicadores assistenciais do CPN Dr. David Capistrano da Costa Filho, em Belo Horizonte, não se distanciam dos resultados apontados na literatura disponível. A baixa taxa de cesárea surge, talvez, como o resultado mais significativo. A modalidade intra-hospitalar mostra-se vantajosa em vários aspectos e eleva a resolutividade do serviço. Contudo, estudos comparativos e controlados que focalizem a assistência hospitalar e a assistência em CPN no Brasil tornam-se necessários para a obtenção de resultados conclusivos. 


\section{Resumo}

A qualidade da assistência prestada em Centro de Parto Normal (CPN) por enfermeira obstetra é amplamente questionada. Foi realizado um estudo descritivo e retrospectivo de 2.117 partos ocorridos entre janeiro de 2002 e julho 2003, no CPN Dr. David Capistrano da Costa Filho, em Belo Horizonte. Entre os principais resultados da assistência, destacam-se a taxa de transferência materna com 11,4\%; a taxa de cesárea com 2,2\%; a taxa de admissão em Centro de Tratamento Intensivo (CTI) neonatal de 1,2\%; e a taxa de Apgar < 7 no 5 o minuto de 1\%. Distocias de trabalho de parto e o desejo por analgesia peridural foram as maiores causas para a transferência materna, enquanto o distúrbio de desconforto respiratório foi a causa principal para admissão dos recém-nascidos no CTI. A mortalidade neonatal corrigida foi de 2 casos em mil nascidos vivos. Percebe-se que os resultados do CPN em estudo não diferem dos dados referidos na literatura internacional. A baixa taxa de cesárea é talvez o resultado mais evidente. Estudos comparativos nacionais são necessários.

Assistência Perinatal; Enfermagem Obstétrica; Parto

\section{Colaboradores}

S. E. V. Campos elaborou o projeto de pesquisa, realizou a coleta e análise dos dados e fez a redação final. F. C. F. Lana contribuiu durante a elaboração e realização do estudo.

\section{Referências}

1. Brasil. Portaria no. 985. Implementa e regulamenta o funcionamento dos Centros de Parto Normal em âmbito do SUS. Diário Oficial da União 1999; 6 ago.

2. Organização Mundial da Saúde. Maternidade segura: atenção ao nascimento normal: guia prático. Genebra: Organização Mundial da Saúde; 1997.

3. Lubic RW, Ernst EKM. The childbearing center: an alternative to conventional care. Nurs Outlook 1978; 26:754-60.

4. Spitzer M. Birth centers: economy, safety and empowerment. J Nurse Midwifery 1995; 40:371-5.

5. Waldenstoem U, Lawson J. Birth centre practices in Australia. Aust N Z J Obstet Gynaecol 1998; 38:42-50

6. Campbell R, MacFarlane A, Hempsall V, Hatchard $\mathrm{K}$. Evaluation of midwife-led care provided at the Royal Bournemouth Hospital. Midwifery 1999; 15:183-93.
7. David M, von Schwarzenfeld HK, Dimer JA, Kentenich H. Perinatal outcome in hospital and birth center obstetric care. Int J Gynaecol Obstet 1999; 65:149-56.

8. Fraser W, Hatem-Asmar M, Krauss I, Maillard F, Breart G, Blais R. Comparison of midwifery care to medical care in hospitals in the Quebec pilot projects study: clinical indicators. L'Equiper dEvaluation des Projets Pilote Sages-Femmes. Can J Public Health 2000; 91:I5-11.

9. Garite TJ, Snell J, Walker DL, Darrow VC. Development and experience of a university-based, freestanding birthing center. Obstet Gynecol 1995; 86:411-6.

10. Greulich B, Paine LL, McClain C, Barger MK, Edwards N, Paul R. Twelve years and more than 30,000 nurse-midwife-attended births: the Los Angeles County + University of Southern California Women's Hospital Birth Center Experience. J Nurse Midwifery 1994; 39:185-96. 
11. Holt J, Vold IN, Backe B, Johansen MV, Qian P. Child birth in a modified midwife managed unit: selection and transfer according to intended place of delivery. Acta Obstet Gynecol Scand 2001; 80:206-12.

12. Hundley VA, Cruickshank FM, Lang GD, Glazener CMA, Milne JM, Turner M, et al. Midwife managed delivery unit: a randomised controlled comparison with consultant led care. BMJ 1994; 309: 1400-4.

13. Jackson DJ, Lang JM, Swartz WH, Ganiats TG, Fullerton J, Ecker J, et al. Outcomes, safety, and resource utilization in a collaborative care birth center program Compared with traditional physician-based perinatal care. Am J Public Health 2003; 93:999-1006.

14. MacVicar J, Dobbie G, Owen-Johnstone L, Jagger C, Hopkins M, Kennedy J. Simulated home delivery in hospital: a randomised controlled trial. $\mathrm{Br} \mathrm{J}$ Obstet Gynaecol 1993; 100:316-23.

15. Rooks JP, Weatherby NL, Ernst EKM, Stapleton S, Rosen D, Rosenfield A. Outcomes of care in birth centers; the National Birth Center Study. N Engl J Med 1989; 32:1804-11.

16. Schmidt N, Abelsen B, Qian P. Deliveries in maternity homes in Norway: results from a 2 year prospective study. Acta Obstet Gynecol Scand 2002; 81:731-7.

17. Waldenstroem U, Nilsson CA, Winbladh B. The Stockholm birth centre trial: maternal e infant outcome. Br J Obstet Gynaecol 1997; 104:410-8.

18. Hodnett ED. Home-like versus conventional institutional settings for birth (Cochrane Review) In: The Cochrane Library, Issue 2, 2003. Oxford: Update Software.
19. Brasil. Lei no. 7498. Dispõe sobre o exercício da enfermagem e dá outras providências. Diário Oficial da União 1986; 26 jun.

20. Ministério da Saúde. Parto, aborto e puerpério: assistência humanizada à saúde. Brasília: Ministério da Saúde/Federação Brasileira das Associações de Ginecologia e Obstetrícia/Associação Brasileira de Obstetrizes e Enfermeiros Obstetras; 2003.

21. Ernst EKM. Midwifery, birth centers, and health care reform. J Obstet Gynecol Neonatal Nurs 1996; 25:433-9.

22. Schwarcz R, Diáz AG, Fescina R, Belitzky R, Mucio B, Delgado L, et al. Saúde reprodutiva materna perinatal: atenção pré-natal e do parto de baixo risco. Montevideo: Centro Latino-Americano de Perinatologia e Desenvolvimento Humano, Organização Pan-Americana da Saúde/Organização Mundial da Saúde; 1996.

23. World Health Organization. Appropriate technology for birth. Lancet 1985; 2:436-7.

24. Hodnett ED. Continuity of caregivers for care during pregnancy and childbirth (Cochrane Review) In: The Cochrane Library, Issue 3, 2003. Oxford: Update Software.

25. Page L, McCourt C, Beake S, Vail A, Hewison J. Clinical interventions and outcomes of One-toOne midwife practice. J Public Health Med 1999; 21:243-8.

26. Cunningham FG, MacDonald PC, Gant NF, Leveno KJ, Gilstrap LC, Hankins GDV, et al. William obstetrícia. Rio de Janeiro: Editora Guanabara Koogan; 2000.

Recebido em 06/Jan/2005

Versão final reapresentada em 22/Dez/2006

Aprovado em 08/Jan/2007 